\title{
BMJ Open Instruments that measure psychosocial factors related to vaccination: a scoping review protocol
}

\author{
Jessica Kaufman (D) , ${ }^{1}$ Rebecca Ryan, ${ }^{1}$ Cornelia Betsch (i),${ }^{2}$ Anne Parkhill, ${ }^{1}$ \\ Gilla Shapiro, ${ }^{3}$ Julie Leask, ${ }^{4,5}$ Lisa Menning, ${ }^{6}$ Peter Tugwell, ${ }^{7}$ Daniel SJ Costa, ${ }^{8}$ \\ Margaret Danchin, ${ }^{9}$ Gabriel Rada, ${ }^{10,11}$ Sophie Hill ${ }^{1}$
}

To cite: Kaufman J, Ryan R, Betsch C, et al. Instruments that measure psychosocial factors related to vaccination: a scoping review protocol. BMJ Open 2019;9:e033938. doi:10.1136/ bmjopen-2019-033938

- Prepublication history and additional material for this paper are available online. To view these files, please visit the journal online (http://dx.doi. org/10.1136/bmjopen-2019033938).

Received 29 August 2019 Revised 16 November 2019 Accepted 18 November 2019

Check for updates

(c) Author(s) (or their employer(s)) 2019. Re-use permitted under CC BY-NC. No commercial re-use. See rights and permissions. Published by BMJ.

For numbered affiliations see end of article.

Correspondence to

Dr Jessica Kaufman;

j.kaufman@latrobe.edu.au

\section{ABSTRACT}

Introduction As vaccine-preventable disease outbreaks increase, there is growing international interest in monitoring public attitudes towards vaccination and implementing and evaluating vaccine promotion interventions. Outcome selection and measurement are central to intervention evaluation. Measuring uptake rates alone cannot determine which elements in a multicomponent vaccine-promotion intervention are most effective, why specific populations are undervaccinated or when confidence in vaccines is wavering. To develop targeted and cost-effective interventions and policies, it is necessary to measure vaccination-related psychosocial factors such as knowledge, attitudes and aspects of decision-making. This scoping review aims to identify, compare and summarise the properties and validation of instruments for measuring vaccinationrelated psychosocial factors and identify gaps where no instruments exist.

Methods and analysis We will search Medline OVID, Embase OVID, CINAHL and PsycINFO with no date restriction, using a pilot-tested search strategy of terms related to vaccination: knowledge, attitudes, trust, acceptance and decision-making and measurement, psychometric testing or validation. This search will be supplemented with manual search and expert consultation. We will include studies that describe instrument development, adaptation or testing and include evaluation of at least two measurement properties (eg, content, criterion, or construct validity; test-retest reliability; internal consistency; sensitivity; responsiveness). Instruments measuring a vaccination-related psychosocial factor in any population will be included. All studies will be screened by one reviewer, with a sample double-screened to confirm accuracy. Disagreements will be resolved with a third reviewer. Data will be synthesised narratively and through summary tables to chart and compare instrument characteristics such as factors measured, date and/ or location of development or validation, measurement properties evaluated and population.

Ethics and dissemination This scoping review aims to provide an overview of existing instruments and ascertain measurement gaps where no measurement instruments currently exist. The identified instruments will form the basis of an open-access online repository of instruments.

\section{Strengths and limitations of this study}

- This review will be the first to provide an overview of instruments that measure vaccination-related psychosocial factors.

- Due to resource restrictions, one author will screen all studies for inclusion, but a sample will be screened again by a second author to ensure reliability.

- To be included, instruments must have at least two measurement properties evaluated. This may reduce the total number of included studies but should ensure that those that are included have been subjected to more than a cursory test for content validation or internal consistency.

\section{INTRODUCTION}

Outbreaks of vaccine-preventable diseases are a growing international crisis, with worldwide measles cases increasing by $300 \%$ from 2018 to 2019 . $^{1}$ Undervaccination and nonvaccination are driven by barriers to access and vaccine hesitancy, with the WHO naming the latter as a threat to global health. ${ }^{23}$ Now, more than ever, there is urgent global focus on the development, implementation and evaluation of interventions and policies to increase vaccine uptake.

Vaccine uptake-like other health behaviours-is shaped by communication, interaction and psychosocial factors. ${ }^{45}$ The language around these factors can vary depending on the context or discipline. Building on our earlier taxonomic work in this area, we consider 'psychosocial factors' to include knowledge, attitudes, values, selfefficacy, vaccine confidence, trust and aspects of individual decision-making. ${ }^{4}$ We also use the term 'factors' here, though they may be referred to as 'constructs' in a psychometric context, or 'outcomes' in intervention evaluation. 
A variety of theoretical models aim to describe the ways in which these psychosocial factors mediate the impact of communication and other interventions on health behaviour. For example, the Health Belief Model, Theory of Planned Behaviour and Social Cognitive Theory highlight the interplay between specific factors including selfefficacy, perceived risks and benefits, attitudes, beliefs, subjective norms and knowledge. ${ }^{6-8}$ Models of shared decision-making also suggest that health behaviour can be shaped by the decision-making experience itself, through quantifiable factors such as anticipated regret, decisional conflict or satisfaction with the process. ${ }^{4}$ For vaccination specifically, additional factors like confidence, trust and values have been shown to be linked to behaviour. ${ }^{9-11}$

Vaccine uptake is generally the ultimate goal of public health policy and intervention. However, understanding and being able to measure psychosocial factors are important at every level, from governments monitoring population health and evaluating interventions to clinicians tailoring communication approaches. For instance, measuring vaccine uptake rates alone cannot establish an intervention's mechanism of effect, or tell us which elements in a multicomponent intervention are most effective and which are unnecessary or even harmful. ${ }^{12} 13$ Along with assessment of practical barriers, measuring vaccination-related psychosocial factors is also necessary to identify target populations, determine potential reasons for undervaccination and inform the design of tailored and cost-effective interventions. ${ }^{14}$ Uptake rates are insufficient for monitoring public sentiment towards vaccination-to detect worrying trends before they become vaccine refusal, regular monitoring of factors like vaccine confidence and trust is required. ${ }^{11} 15$ Finally, ensuring that people are informed, supported and satisfied with their healthcare decision-making experiences is an ethical and human rights imperative that can also be quantifiably assessed. ${ }^{16}$

Despite the importance of these psychosocial factors in explaining or shaping vaccine-uptake behaviour, they are frequently overlooked in intervention, policy and programme evaluations. ${ }^{17}$ Evaluations may not have sufficient resources or expertise to incorporate such additional measures, evaluators may not be aware of or may not value additional measures, or there may be no easily-accessible or identifiable instruments with which to measure a concept. Even when these additional psychosocial factors are measured, it is often with instruments that are developed ad-hoc, incompletely validated or used only once. These instruments are then difficult to find, interpret or apply in future studies. Previous reviews of the effect of communication interventions at both individual and community levels found significant heterogeneity in outcome measures, indicating the need for greater standardisation to enable better comparison. ${ }^{18} 19$ A selection of seven vaccine acceptance or hesitancy measures has been briefly summarised elsewhere. ${ }^{10}$ However, there is no broad overview of the instruments available for measuring the full range of psychosocial factors related to vaccination, including knowledge, attitudes, values, selfefficacy, vaccine confidence, trust and decision-making.

Therefore, this systematic scoping review aims to (a) identify, compare and summarise the properties and validation of instruments to measure vaccination-related psychosocial factors and (b) identify gaps in the factors for which instruments exist.

\section{METHODS AND ANALYSIS}

This scoping review will apply the framework developed by Arksey and O'Malley and further expanded by the Joanna Briggs Institute. ${ }^{2021}$ This framework involves the following stages: define the review aim and eligibility criteria, identify relevant studies, screen and select studies, extract and chart the data and collate and summarise the results. The initial stages, through study selection, will also draw from the COSMIN methodology for systematic reviews of Patient-Reported Outcome Measures. ${ }^{22}$ Reporting will follow the Preferred Reporting Items for Systematic Reviews and Meta-Analyses extension for Scoping Reviews checklist. ${ }^{23}$ The estimated review completion date is 31 March 2020.

\section{Eligibility criteria}

Studies meeting the following criteria will be included:

- Study type: any published studies which aim to (1) develop a new instrument OR (2) adapt, translate or test an existing instrument in a new population; AND (3) evaluate at least two measurement properties (eg, content validity, criterion validity, construct validity, test-retest reliability, internal consistency, predictive validity, sensitivity, or responsiveness). If a study tests only one measurement property but another study tests one or more other properties of the same instrument, these will be included. Face validity is not considered a relevant measurement property for the purposes of determining study inclusion because it is a subjective judgement made by individuals with no expertise in the subject area. ${ }^{24}$

- Factors: the instrument measures one or more psychosocial factors relevant to vaccination, including but not limited to knowledge, attitudes, values, selfefficacy, vaccine confidence, trust and aspects of individual decision-making.

- Population: any population eligible for vaccination or responsible for making vaccination decisions for others (including healthcare workers, students, parents, adolescents, children, pregnant women and elderly people).

There will be no date, location or language restrictions in our search. Where studies published in languages other than English are identified, we will use freely-available online translation tools to enable screening for relevance. We will contact study authors and/or seek full translation for relevant non-English studies if resources allow.

\section{Search strategy}

We searched the following electronic databases from inception to August 7, 2019: Medline OVID, Embase 
OVID, CINAHL and PsycINFO. The search strategy includes index and text words related to vaccination or immunisation, knowledge, attitudes, acceptance and decision-making and measurement, psychometric testing or validation. Key MeSH terms were drawn from a framework of vaccination communication outcome domains (ie, knowledge, attitudes, decision-making). ${ }^{4}$ Specific search keywords were identified from titles, keywords and abstracts of a sample of approximately 50 previously identified relevant studies across the range of psychosocial factors. Using these relevant studies, we pilot- tested and refined the search strategy to ensure it will be both sensitive and focused. The full search strategy is included as an appendix (online supplementary additional file 1).

We will also review the reference lists of relevant studies and consult experts in the field, identified through the authors' international networks, to identify any additional references or links to instruments.

\section{Study selection}

Search results will be loaded into Endnote X8 (Clarivate Analytics 2016) and duplicates will be removed. Studies will be screened first by title and abstract, with potentially eligible studies screened by full text. One author (JK) will conduct the primary screening. A second author will screen a sample of the results to compare and confirm the screening approach. Other authors will be asked to provide input where screening decisions are not straightforward.

\section{Data extraction}

One review author (JK) will extract data from all included studies, using a standardised data extraction template developed for this review (online supplementary additional file 2). The extracted data will include:

- Citation details (eg, title, year of publication, authors).

- Type of study (instrument development and initial validation, validation of adapted or translated instrument, validation in a new population).

- Instrument title and/or abbreviation.

- Summary of instrument topic/purpose (eg, 'parental decision-making about human papillomavirus vaccine for their daughters').

- Subscales, including definition provided by the authors and number of items per subscale.

- Number of total items.

- Ranges of scores/scoring description.

- Population of intended use.

- Population of validation. (Sample size, age, gender, setting, country, language).

- Country of initial development.

- Available languages.

- Accessibility (location/cost).

- Linked references (ie, other validation studies related to this tool or other versions of the tool).

- Measurement properties evaluated (content validation, criterion validation, construct validation, test-retest reliability, internal consistency, predictive validation, sensitivity and responsiveness).

Any uncertainties, for example, about the nature of measurement property assessment, will be raised with the other authors for discussion and resolution.

In keeping with standard scoping review methodology, we will assess the degree of validation for each tool by reporting which measurement properties have been evaluated, but will not appraise the quality of the specific validation methods used. ${ }^{23} 25$

\section{Data synthesis strategy}

The data will be synthesised narratively and through summary tables which will chart the characteristics of the instruments for ease of comparison. The synthesis will provide an overview of the instruments measuring each factor, with specific subanalyses organised by relevant features such as date and/or location of development or validation and population of intended use. Comparative tables will be used where relevant. For each tool, we will summarise the measurement properties evaluated using tables similar to the Cochrane risk of bias summary figures for intervention reviews. ${ }^{26}$ If many instruments measuring the same factors are identified, their key differences and similarities will be explored with more detailed analysis. To identify gaps, that is, factors that are not measured in any identified instruments, the instruments will be mapped against a taxonomy of outcomes relevant to vaccination communication. ${ }^{4}$

\section{Patient and public involvement}

The development of this scoping review protocol did not involve patients or the public.

\section{ETHICS AND DISSEMINATION}

This scoping review is intended to help researchers, policymakers, vaccination programme officials and other stakeholders identify appropriate, fit-for-purpose instruments to gather population data and evaluate vaccine promotion strategies. Healthcare practitioners may also find useful instruments to apply as waiting-room screening tools to determine people's potential vaccine hesitancy, knowledge or values and inform their communication strategies. This review will also highlight gaps where there are no available instruments to measure specific factors. There are no ethical considerations related to this review.

The results of this review will form the basis of an open access online repository of instruments to be developed through the Measurement Outcomes for Vaccination Evaluations project. ${ }^{27}$

\section{Author affiliations}

${ }^{1}$ Centre for Health Communication and Participation, School of Psychology and Public Health, La Trobe University, Bundoora, Victoria, Australia

${ }^{2}$ Health Communication, Faculty of Philosophy, University of Erfurt, Erfurt, Germany ${ }^{3}$ Department of Supportive Care, Princess Margaret Cancer Centre, University Health Network, Toronto, Ontario, Canada

${ }^{4}$ School of Public Health, University of Sydney, Camperdown, New South Wales, Australia 
${ }^{5}$ Faculty of Nursing, University of Sydney, Camperdown, New South Wales, Australia ${ }^{6}$ Immunization, Vaccines and Biologicals, World Health Organization, Geneve, GE, Switzerland

${ }^{7}$ Medicine, University of Ottawa, Ottawa, Ontario, Canada

${ }^{8}$ Pain Management Research Institute, The University of Sydney, St Leonards, New South Wales, Australia

${ }^{9}$ Vaccine and Immunisation Research Group, Murdoch Childrens Research Institute, Parkville, Victoria, Australia

${ }^{10}$ Evidence-Based Healthcare Program, Pontificia Universidad Católica de Chile, Santiago, Chile

${ }^{11}$ Epistemonikos, Santiago, Chile

\section{Twitter Gabriel Rada @radagabrie}

Acknowledgements The authors would like to thank Lina Schonfeld for helping to pilot the data extraction form and Steve McDonald for contributing to early discussions of the review and Measurement Outcomes for Vaccination Evaluations project.

Contributors JK, RR, CB, AP, JL, LM, PT, DSJC, MD, GR and SH discussed scope and content of the review in planning stages, as a part of the Measurement Outcomes for Vaccination Evaluations project. JK drafted the manuscript. AP developed and helped refine the search strategy. CB, GS and DC provided specific feedback on psychometric testing issues. JK, RR, CB, GS, AP, JL, LM, PT, DSJC, MD, GR and SH provided feedback on drafts and approved the final manuscript.

Funding Salary support for JK is provided by the Centre for Health Communication and Participation at La Trobe University.

Competing interests None declared.

Patient consent for publication Not required.

Provenance and peer review Not commissioned; externally peer reviewed.

Open access This is an open access article distributed in accordance with the Creative Commons Attribution Non Commercial (CC BY-NC 4.0) license, which permits others to distribute, remix, adapt, build upon this work non-commercially, and license their derivative works on different terms, provided the original work is properly cited, appropriate credit is given, any changes made indicated, and the use is non-commercial. See: http://creativecommons.org/licenses/by-nc/4.0/.

\section{ORCID iDs}

Jessica Kaufman http://orcid.org/0000-0001-5139-4183

Cornelia Betsch http://orcid.org/0000-0002-2856-7303

\section{REFERENCES}

1 Mahase E. Measles cases rise $300 \%$ globally in first few months of 2019. BMJ 2019.

2 Omer SB, Betsch C, Leask J. Mandate vaccination with care. Nature 2019;571:469-72.

3 World Health Organization. Ten threats to global health in 2019, 2019. Available: https://www.who.int/emergencies/ten-threats-to-globalhealth-in-2019

4 Kaufman J, Ryan R, Glenton C, et al. Childhood vaccination communication outcomes unpacked and organized in a taxonomy to facilitate core outcome establishment. $J$ Clin Epidemiol 2017;84:173-84.

5 Brewer NT, Chapman GB, Rothman AJ, et al. Increasing vaccination: putting psychological science into action. Psychol Sci Public Interest 2017;18:149-207.
6 Ajzen I. From Intentions to Actions: A Theory of Planned Behavior. In: Kuhl J, Beckmann J, eds. Action control: from cognition to behavior. Berlin: Springer-Verlag, 1985: 11-39.

7 Bandura A. Social foundations of thought and action: a social cognitive theory. Englewood cliffs. NJ: Prentice-Hall, 1986.

8 Janz NK, Becker MH. The health belief model: a decade later. Health Educ Q 1984;11:1-47.

9 Amin AB, Bednarczyk RA, Ray CE, et al. Association of moral values with vaccine hesitancy. Nat Hum Behav 2017;1:873-80.

10 Betsch C, Schmid P, Heinemeier D, et al. Beyond confidence: development of a measure assessing the $5 \mathrm{C}$ psychological antecedents of vaccination. PLoS One 2018:13:e0208601.

11 Larson $\mathrm{HJ}$, Clarke RM, Jarrett $\mathrm{C}$, et al. Measuring trust in vaccination: a systematic review. Hum Vaccin Immunother 2018;14:1599-609.

12 Breuer $\mathrm{E}$, Lee L, De Silva M, et al. Using theory of change to design and evaluate public health interventions: a systematic review. Implementation Sci 2015;11.

13 Michie S, Carey RN, Johnston M, et al. From Theory-Inspired to Theory-Based interventions: a protocol for developing and testing a methodology for linking behaviour change techniques to theoretical mechanisms of action. Ann Behav Med 2018;52:501-12.

14 Butler R, MacDonald NE, SWGoV H. Diagnosing the determinants of vaccine hesitancy in specific subgroups: the guide to tailoring immunization programmes (tip). Vaccine 2015;33:4176-9.

15 Widdus $\mathrm{R}$, Larson $\mathrm{H}$, mandates V. Vaccine mandates, public trust, and vaccine confidence: understanding perceptions is important. $J$ Public Health Policy 2018;39:170-2.

16 Hill S, Draper M. A new conceptual framework for advancing evidence-informed communication and participation. In: Hill S, ed. The Knowledgeable patient: communication and participation in health. UK: Wiley Blackwell, 2011: 12-26.

17 Kaufman J, Ryan R, Bosch-Capblanch X, et al. Outcomes mapping study for childhood vaccination communication: too few concepts were measured in too many ways. J Clin Epidemiol 2016;72:33-44.

18 Kaufman J, Ryan R, Walsh L, et al. Face-To-Face interventions for informing or educating parents about early childhood vaccination. Cochrane Database Syst Rev 2018;5.

19 Saeterdal I, Lewin S, Austvoll-Dahlgren A, et al. Interventions aimed at communities to inform and/or educate about early childhood vaccination. Cochrane Database Syst Rev 2014;11.

20 Arksey H, O'Malley L. Scoping studies: towards a methodological framework. Int J Soc Res Methodol 2005;8:19-32.

21 Peters MDJ, Godfrey C, Mclnerney P, et al. Chapter 11: Scoping Reviews. In: Aromataris E, M Z, eds. Joanna Briggs Institute reviewer's manual: the Joanna Briggs Institute, 2017.

22 Mokkink LB, Prinsen CA, Patrick DL, et al. COSMIN methodology for systematic reviews of Patient-Reported outcome measures (PROMs): user manual: COSMIN, 2018. Available: https://www.cosmin.nl/wpcontent/uploads/COSMIN-syst-review-for-PROMs-manual_version1_feb-2018-1.pdf

23 Tricco AC, Lillie E, Zarin W, et al. PRISMA extension for scoping reviews (PRISMA-ScR): checklist and explanation. Ann Intern Med 2018;169:467-73.

24 Holden RR, Validity FWeiner IB, Craighead WE, eds. The Corsini encyclopedia of psychology. John Wiley \& Sons, Inc, 2010.

25 Peters MDJ, Godfrey CM, Khalil H, et al. Guidance for conducting systematic scoping reviews. Int J Evid Based Healthc 2015;13:141-6.

26 Higgins JPT, Altman DG, Sterne JAC. 8.6 Presentation of assessments of risk of bias. 2011. In: Cochrane Handbook for Systematic Reviews of Interventions [Internet]. The Cochrane Collaboration. Available: www.handbook.cochrane.org

27 Centre for Health Communication and Participation. Measurement outcomes for vaccination evaluations (move): La Trobe university, 2019. Available: https://www.latrobe.edu.au/chcp/projects/move 